\title{
Cytokine release syndrome as an important differential diagnosis of severe skin toxicity with organ damage during switch from immunotherapy to targeted therapy in metastatic melanoma
}

Dimitriou, Florentia ; Mangana, Joanna ; Micaletto, Sara ; Braun, Ralph P ; Dummer, Reinhard

DOI: https://doi.org/10.1097/CMR.0000000000000544

Posted at the Zurich Open Repository and Archive, University of Zurich ZORA URL: https://doi.org/10.5167/uzh-160597

Journal Article

Published Version

Originally published at:

Dimitriou, Florentia; Mangana, Joanna; Micaletto, Sara; Braun, Ralph P; Dummer, Reinhard (2019). Cytokine release syndrome as an important differential diagnosis of severe skin toxicity with organ damage during switch from immunotherapy to targeted therapy in metastatic melanoma. Melanoma research, 29(1):107-108.

DOI: https://doi.org/10.1097/CMR.0000000000000544 


\section{Letter to the Editor}

\section{Cytokine release syndrome as an important differential diagnosis of severe skin toxicity with organ damage during switch from immunotherapy to targeted therapy in metastatic melanoma}

Florentia Dimitriou, Joanna Mangana, Sara Micaletto,

Ralph P. Braun and Reinhard Dummer, Department of Dermatology, University Hospital of Zurich, Zurich, Switzerland

Correspondence to Reinhard Dummer, MD, Department of Dermatology, University Hospital of Zurich, Gloriastrasse 31, 8091 Zurich, Switzerland Tel: + 41442552 507; fax: + 41442553 999; e-mail: reinhard.dummer@usz.ch

Received 21 September 2018 Accepted 9 October 2018

Lamiaux and colleagues [1] found that $19 \%$ of the patients treated with BRAF/MEK inhibitors (BRAFi/ MEKi) after previous immunotherapy (IT) may present a severe skin rash (grade $\geq 3$ ), even complicated by systemic involvement and organ damage. Among the 10 cases of severe skin toxicity, eight patients had received previous IT, whereas the median time of onset was 12 days after the treatment initiation of BRAFi/MEKi. In four patients with kidney, liver, and heart involvement, the symptoms were interpreted as part of a systemic reaction [drug rash with eosinophilia, and systemic symptoms (DRESS)]; yet, the DRESS criteria were only partially fulfilled and no peripheral eosinophilia was reported. Patients were treated with local and intravenous steroids. The systemic treatment with BRAFi/ MEKi was, in most of the cases, discontinued permanently.

We have previously reported four cases of cytokine release syndrome (CRS) during the sequential use of BRAFi/MEKi after immune checkpoint blockade with severe (grade $\geq 3$ ) skin and systemic involvement [2,3]. The constellation of the symptoms described in our cases, which included among all and except for pyrexia and maculopapular rash also a transaminitis, an uncommon event during treatment with BRAFi/MEKi, fulfills the diagnosis of a CRS. The documented, high circulating cytokine levels, including interleukin-6 (IL-6), tumor necrosis factor- $\alpha$, and interferon- $\gamma$, further confirm our hypothesis. These clinical cases underline the complexity of the immunologic interactions caused during the sequential therapy with immune checkpoint inhibitors and kinase inhibitors. These manifestations should be distinguished from a hypersensitivity syndrome to the kinase inhibitors (DRESS); the lack of the frequently observed eosinophilia of DRESS as well as other blood abnormalities, reactivation of human herpes virus 6 , or lymphadenopathy do not support the diagnosis. The short onset of the symptoms after the treatment initiation (in our cases, the median time was 10 days) and the tolerance of the identical medication after reinduction are also typical for a systemic inflammatory reaction and untypical for a DRESS. Besides, a lymphocyte transformation test performed on one of our patients was negative. We attribute this systemic reaction to an aberrant release of proinflammatory cytokines, mainly accompanied by symptoms such as pyrexia, transaminitis, renal insufficiency, and maculopapular skin rash. Thus, such episodes have also been reported in the preliminary findings of a phase III clinical trial with dabrafenib, trametinib, and anti-PD1 (NCT02967692) [4].

We suggest a 'cytokine storm' as a possible mechanism of the CRS because of a high level of immune activation in the context of the simultaneous presence of immune checkpoint and MAPK pathway inhibition. Targeting oncogenic drivers in the immune system leads to a T-cell activation and consecutive release of cytokines, such as tumor necrosis factor- $\alpha$ and interferon- $\gamma$, with further activation of innate immune cells and production of numerous cytokines, among all also IL-6, which importantly causes a positive feedback loop, leading to a 'cytokine storm' [5]. However, clinical and preclinical data propose that MAPK pathway inhibition may enhance T-cell activation through an increase of proinflammatory cytokines and cytotoxic factors [6]. These changes to the tumor microenvironment would be expected to increase efficacy by making tumors more susceptible to IT.

We therefore propose CRS as an important and severe differential diagnosis in patients with severe rash and systemic symptoms during the sequential treatment of targeted therapy and IT. The clear description of our cases along with the successful use of tocilizumab (antiIL-6 agent) suggests that CRS is a severe manifestation occurring early during the initiation of targeted therapy after the treatment of IT in patients with metastatic melanoma. As the management and treatment of the CRS can be very challenging, we advise the practitioners to measure the cytokine levels and use cytokine antagonists as potential therapeutic candidates in cases refractory to steroids. 


\section{Acknowledgements Conflicts of interest}

R.D. has intermittent, project-focused consulting and/or advisory relationships with Novartis, Merck Sharp \& Dhome, Bristol-Myers Squibb, Roche, Amgen, Takeda, and Pierre Fabre outside the submitted work. J.M. has a temporary advisory relationship and receives travel support from Pierre Fabre, Merck Sharp \& Dhome, and Merck outside the submitted work. For the remaining authors, there are no conflicts of interest.

\section{References}

1 Lamiaux M, Scalbert C, Lepesant P, Desmedt E, Templier C, Dziwniel V, et al. Severe skin toxicity with organ damage under the combination of targeted therapy following immunotherapy in metastatic melanoma. Melanoma Res 2018; 28:451-457.
2 Dimitriou F, Matter AV, Mangana J, Urosevic-Maiwald M, Micaletto S, Braun RP, et al. Cytokine release syndrome during sequential treatment with immune checkpoint inhibitors and kinase inhibitors for metastatic melanoma. J Immunother 2018. [Epub ahead of print].

3 Urosevic-Maiwald M, Mangana J, Dummer R. Systemic inflammatory reaction syndrome during combined kinase inhibitor therapy following anti-PD-1 therapy for melanoma. Ann Oncol 2017; 28:1673-1675.

4 Dummer R, Arance Fernández AM, Hansson J, Larkin JMG, Long GV, Gasal E, et al. Preliminary findings from part 1 of COMBI-i: a phase III study of anti-PD1 antibody PDR001 combined with dabrafenib (D) and trametinib (T) in previously untreated patients (pts) with advanced BRAF V600-mutant melanoma. J Clin Oncol 2018; 36 (Suppl):189.

5 Shimabukuro-Vornhagen A, Godel P, Subklewe M, Stemmler HJ, Schlosser HA, Schlaak M, et al. Cytokine release syndrome. J Immunother Cancer 2018; 6:56.

6 Frederick DT, Piris A, Cogdill AP, Cooper ZA, Lezcano C, Ferrone CR, et al. BRAF inhibition is associated with enhanced melanoma antigen expression and a more favorable tumor microenvironment in patients with metastatic melanoma. Clin Cancer Res 2013; 19:1225-1231. 